\title{
IXODES SIGALASI N. SP., IXODOIDE NOUVEAU DES OISEAUX
}

\author{
Par M. LAMONTELLERIE
}

Au cours de nos recherches sur les Ixodoïdes du Sud-Ouest de la France, nous nous sommes procuré deux exemplaires femelles d'Ixodes que nous rapportons à une espèce nouvelle; nous dédions cette espèce à notre Maître, M. le professeur Sigalas, et nous proposons de la nommer lxodes sigalasi n. sp.

Nous avions tout d'abord rapporté ces deux exemplaires à l'espèce Ixodes frontalis Panzer, mais les travaux de D. R. Arthur sur les espèces voisines d'Ixodes frontalis Panzer (Ixodes brunneus Koch, Ix. daveyi Nuttall, Ix. theileri Arthur), nous ont fait reconsidérer la question. L'étude morphologique de ces deux exemplaires nous a permis d'y découvrir un certain nombre de caractères permettant de les distinguer des quatre espèces précitées, et nous nous proposons d'en donner la description, ainsi que les principaux caractères permettant de différencier cette forme nouvelle des espèces voisines.

Origine de ces deux individus. - L'exemplaire $\mathrm{n}^{\circ} 1$ a été capturé à Montendre (Charente-Maritime), le 15 janvier 1953, sur un vanneau (Vannellus vannellus $\mathrm{L}$.). L'exemplaire $\mathrm{n}^{\circ} 2$ a été trouvé fixć sur la nuque d'une grive (Turdus sp.), dont la détermination spécifique précise n'a pu être faite (l'oiseau ayant été plumé et mangé lorsque nous fut apporté son parasite). La grive'a été tuée aux environs de Dax (Landes), le 8 février 1954.

Description de l'exemplaire $\mathrm{n}^{* 1} \mathbf{1}$ (photo). - Il s'agit d'une femelle non gorgée qui était morte à son arrivée au laboratoire. Autour de l'hypostome subsistait un fragment des téguments de l'hôte; le tarse IV gauche manque.

Corps ovale, plus large en arrière du milieu, au niveau des spiracles.

Coloration brun foncé, plus marquée au niveau des parties latéAnx. ye Paikastrologie, T. XXIX, n 5-6. - 1954. 
rales du scutum. Portion médiane du scutum brun rouge; pattes et collare brun rougeâtres.

Longueur totale (depuis la pointe de l'hypostome) : $3,17 \mathrm{~mm}$.

Plus grande largeur (au niveau des péritrèmes) : 1,81 mm.

Collare : Face supérieure de forme générale triangulaire, dont la plus grande largeur est de $0,48 \mathrm{~mm}$. (fig. 7).

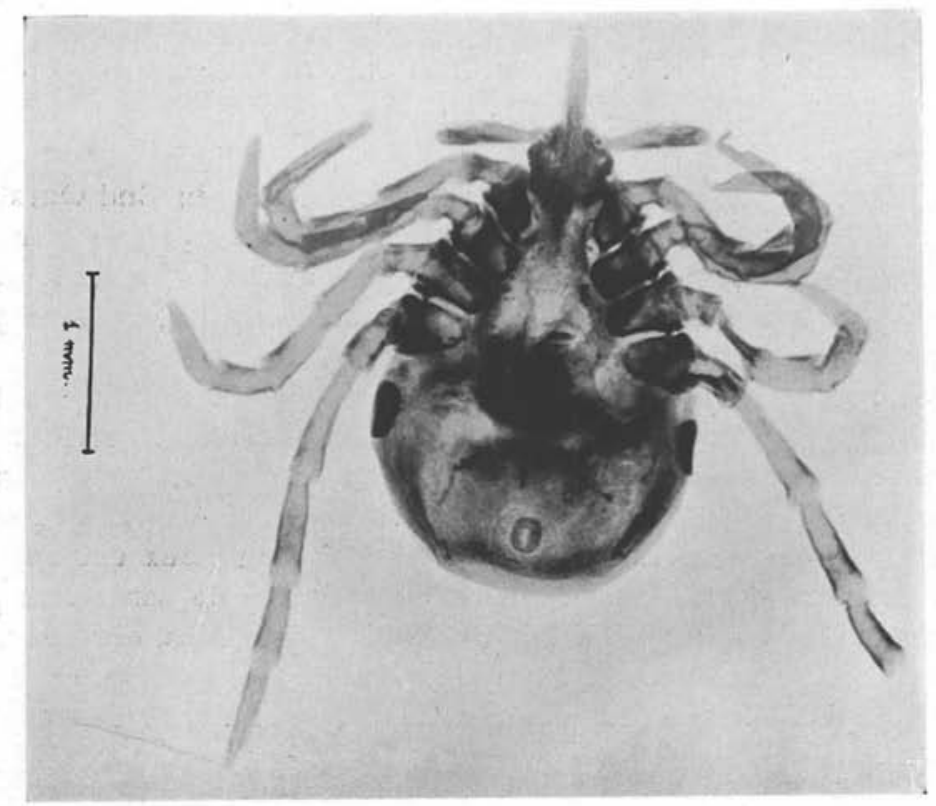

Рното : Ixodes sigalasi n. sp., exemplaire $\mathrm{n}^{\circ} 1$, face vestrale.

Bord latéral convexe, avec une petite incisure à l'union du tiers antérieur et des deux tiers postérieurs.

Bord postérieur légèrement concave, cornua très petites mais nettement marquées.

Aires poreuses ovoïdes transversalement, grandes, s'étendant jusqu'aux bords latéraux et postérieur, séparées par une distance égale au tiers de leur plus grand axe.

Face inférieure de forme générale rectangulaire (fig. 5).

Plus grande longueur : $0,42 \mathrm{~mm}$.

Plus grande largeur : $0,46 \mathrm{~mm}$.

Angles postérieurs arrondis. Un net rétrécissement en arrière des auricules. 
Auricules bien développés, à bord postérieur convexe, à bord antérieur légèrement concave, présentant un angle assez marqué à l'union des bords postérieur et antérieur.

Hypostome : Long $(0,5 \mathrm{~mm}$.), pointu à l'extrémité. Dents réparties suivant la formule : $4 \times 12+3 \times 3+2 \times 2$.

Pas de dents dans l'aire centrale.

Palpes : Longs et étroits, arrondis à l'extrémité. Longueur totale : $0,65 \mathrm{~mm}$. Bord externe sensiblement rectiligne, bord interne convexe.

Article I : Court $(0,12 \mathrm{~mm}$.), plus large que long, présentant une petite épine à la face inférieure.

Article II : Allongé $(0,27 \mathrm{~mm}$.), extrémité proximale rétrécie. Bord interne convexe, bord externe sensiblement rectiligne. Faces supérieure et inférieure portant quelques longues soies, réparties surtout vers les bords interne et externe.

Article III : Allongé $(0,26 \mathrm{~mm}$.). A la face inférieure, en bas et en dedans de l'article IV se trouve un petit bourrelet mousse.

Article IV : Très petit, portant de nombreuses soies.

Scutum (fig. 9) : Plus long que large : longueur 1,3 mm. Largeur maxima : $1,04 \mathrm{~mm}$.

Angles latéraux largement arrondis, bord postérieur arrondi, plus grande largeur située en avant du milieu.

Scapulæ nettes, pointues.

Sillons cervicaux légèrement convergents en avant, divergents en arrière.

L'aire médiane (limitée de chaque côtẻ par les sillons cervicaux), porte une ponctuation double : points fins, épars vers l'avant, plus abondants vers l'arrière, et gros points où s'insèrent de longues soies dirigées en arrière ; dans la région tout antérieure, la cuticule est finement striée transversalement.

Les aires latérales sont plus rugueuses et portent des soies un peu plus développées que celles de l'aire médiane; au niveau des scapulæ, cuticule finement striée.

Alloscutum : Cuticule très striée ; poils longs, recourbés vers l'arrière, nombreux.

Face inférieure : Cuticule très striée, présentant de longues soies recourbées vers l'arrière, insérées sur de gros points. Entre les hanches les points d'insertion des soies sont plus gros et plus nombreux; les soies sont nettement plus développées que dans les autres régions.

Orifice génital : Situé au niveau des coxæ III ; lèvre postérieure triangulaire. 
Uropore (fig. 10) : Sillon anal en fer à cheval, s'arrêtant un peu avant le bord postérieur. Chaque lèvre de l'uropore porte 3 soies dirigées en arrière.
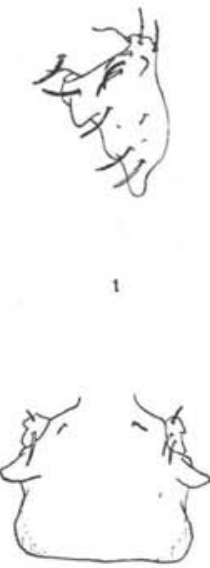

s
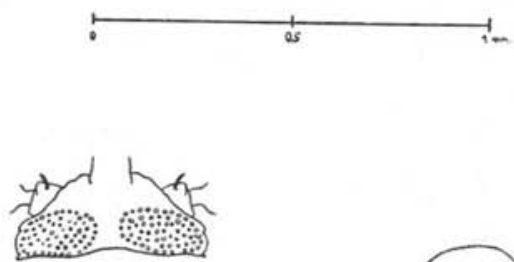

7

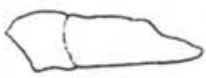

19
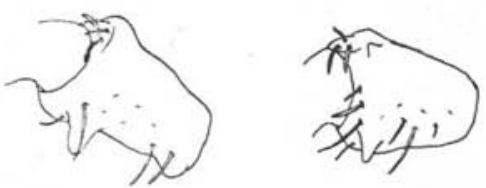

3
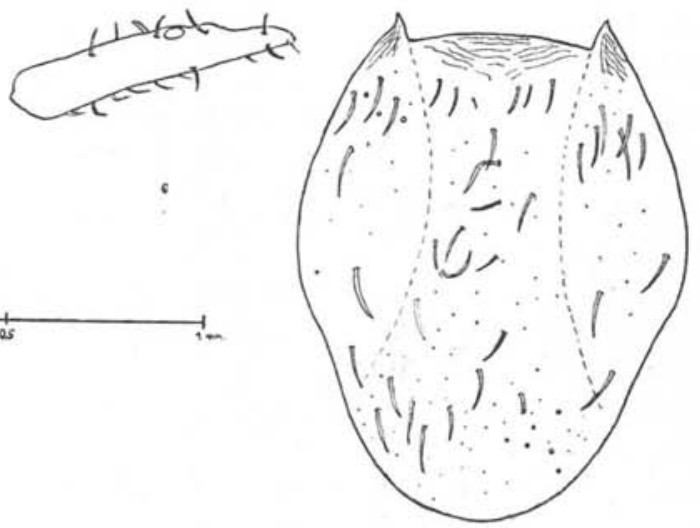

Ixodes sigalasi n. sp., exemplaire $\mathrm{n}^{\circ} 1$ :

Fig. 1 à 4 : Coxae I à IV. - Fig. 5 : Collare, face ventrale. - Fig. 6 : Tarse I. - Fig. 7 : Collare, face dorsale. - Fig. 8: Tarse II. - Fig. 9: Scutum. Fig. 10 : Uropore.

Péritrèmes : Ovoïdes, situés en arrière des coxæ IV (à une distance égale à la moitié de leur longueur). Un peu surélevés; le spiracle un peu excentrique.

Pattes : Coxæ: bien séparées les unes des autres. La coxa I pré- 
sente deux éperons, un interne et un externe, l'interne un peu plus long et plus aigu que l'externe (fig. 1).

Les coxæ II à IV présentent un fort éperon externe, dont la taille décroît de II à IV. Présence d'un bourrelet interne sur les coxæ II et III (fig. 2 à 4 ).

Trochanters : un faible éperon trochantérien, de I à IV. Cet éperon est un peu plus fort aux pattes II et III.

Tarses: longs, en particulier le tarse I $(0,73 \mathrm{~mm}$.$) , qui est de$ longueur triple du métatarse correspondant $(0,24 \mathrm{~mm}$.) (fig. 6).

Les tarses II (long. $0,48 \mathrm{~mm}$.) à IV sont proportionnellement plus courts (fig. 8').

Tous les tarses sont brusquement rétrécis près de leur extrémité distale.

Ambulacres : presque aussi longs que les ongles.

Description de l'exemplaire $\mathrm{n}^{\circ} 2$. - Il s'agit d'une femelle gorgée qui nous a été apportée en état de mort apparente : un séjour de quelques heures en tube d'élevage (technique de Métianu, légèrement modifiée), lui a redonné une certaine vitalité. L'animal est mort trois jours après son arrivée au laboratoire.

Aspect général rappelant celui d'une femelle gorgée d'Ixodes ricinus L., mais à bord postérieur plus rectiligne.

Coloration brun noir; pattes, palpes et collare brun rougeàtres. Le scutum présente une coloration double, aire médiane (limitée par les sillons cervicaux) brun rouge, aires latérales presque noires.

Longueur (depuis la pointe de l'hypostome) $5,8 \mathrm{~mm}$.

Plus grande largeur (en arrière des péritrèmes) $4,2 \mathrm{~mm}$.

La morphologie de cet exemplaire est superposable à celle du précédent, notons cependant les particularités suivantes :

Alloscutum à pilosité rare : les soies sont pour la plupart seetionnées à un niveau variable, ce que nous estimons être imputable au fait qu'il s'agit ici d'un exemplaire ‘́gé. Mème remarque en ce qui concerne la face inférieure.

Comme chez Ixodes frontalis Panzer, on note la présence, dans la moitié postérieure de l'alloscutum, de deux sillons recourbés se réunissant vers le milieu de la longueur en forme de fer à cheval.

Sillons sexuels très divergents vers l'arrière, un peu resserrés vers la partie médiane ; leur extrémité aboutit bien avant le bord postérieur.

Sillon anal à branches sensiblement parallèles dans la partie antérieure, puis convergentes en arrière. Ce sillon se termine bien avant le bord postérieur.

Péritrèmes ovoïdes, très éloignés des coxæ IV. Spiracle excentrique, déporté en bas et en avant. 
Voici quelles sont les principales mensurations de l'animal :

- Collare : longueur $0,43 \mathrm{~mm}$; largeur $0,47 \mathrm{~mm}$.

- Hypostome : longueur $0,56 \mathrm{~mm}$.

- Palpes : longueur totale $0,69 \mathrm{~mm}$. ; $2^{\circ}$ article $0,3 \mathrm{~mm}$. ; $3^{\text {e article }}$ $0,28 \mathrm{~mm}$.

- Scutum : longueur 1,3 mm. ; largeur maxima 1,06 mm.

- Pattes : tarse I $0,68 \mathrm{~mm}$. ; métatarse I $0,22 \mathrm{~mm}$. t tarse II $0,47 \mathrm{~mm}$.

\section{Discussion}

Du point de vue des mensurations générales, Ixodes sigalasi n. sp. se rapproche de $I x$. brunneus et de $I x$. frontalis. Il s'écarte nettement de $I x$. theileri.

Le seul caractère du sillon anal (en fer à cheval), l'écarte de brunneus et de frontalis, mais le fait entrer dans le groupe daveyitheileri.

Il s'éloigne notablement de daveyi, en particulier par les caractères de l'hypostome et la position de l'orifice génital.

Il diffère de theileri en particulier par les caractères principaux suivants :

Ixodes theileri Arthur, 1953

Ixodes sigalasi n.sp.

Angle postérieur de la face inférieure du collare en angle droit. Auricules à bord latéral net.

Bourrelet interne aux hanches II à IV.

Epine trochantérienne de I à III. Taille grande.
Arrondi.

Bords antérieur et postérieur se réunissant angulairement.

Absent à la hanche IV.

De I à IV.

Taille petite.

Il est possible que cette espèce soit à rattacher à celle décrite par Pomerantzev en 1950 sous le nom d'Ixodes frontalis (Panzer), à tort d'ailleurs, puisque cet auteur parle d'une espèce à sillon anal en fer à cheval.

Enfin, il nous semble certain qu'Ixodes sigalasi doit être inféodé aux oiseaux, comme les autres espèces du même groupe, et le fait même de sa découverte sur des oiseaux essentiellement migrateurs indique qu'elle doit avoir une vaste aire de répartition. 
Tableau permettant de séparer les diverses espèces du même groupe :

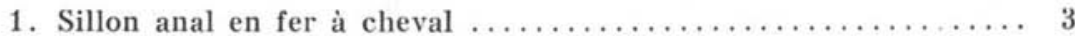

Sillon anal à bords parallèles ou divergents en arrière ....... 2

2. Aires poreuses ovoïdes, grandes ;

tarses graduellement rétrécis ......... Ix. brunneus Koch. Aires poreuses arrondies, petites ;

tarses brusquement rétrécis ......... Ix. frontalis Panzer.

3 . Pore génital au niveau des hanches III ................ 4

Pore génital au niveau des hanches IV ........ Ix. daveyi Nuttall.

4. Collare à angles postéro-inférieurs droits ;

taille grande $\ldots \ldots \ldots \ldots \ldots \ldots \ldots \ldots \ldots \ldots \ldots \ldots \ldots$ theileri Arthur.

Collare à angles postéro-inférieurs arrondis ;

taille petite $\ldots \ldots \ldots \ldots \ldots \ldots \ldots \ldots \ldots \ldots \ldots \ldots \ldots \ldots$ I . sigalasi n.sp.

\section{Conclusions}

Nous présentons deux exemplaires d'un Ixodoïde, hôte d'oiseaux migrateurs, ayant des affinités diverses avec Ixodes frontalis Panzer, Ix. brunneus Koch, Ix. daveyi Nuttall et Ix. theileri Arthur.

Nous proposons pour cette nouvelle espèce le nom d'Ixodes sigalasi.

A l'heure actuelle, les Ixodidés des oiseaux sont encore plus mal connus que ceux des mammifères et nous estimons que la classification que nous utilisons, basée avant tout sur la morphologie, devra être fortement remaniée lorsqu'on connaîtra la biologie et les variations propres à chaque espèce ; un certain nombre d'espèces actuelles pourront alors disparaître et il est possible que le groupe frontalis-brunneus-daveyi-theileri-sigalasi doive être réduit à une ou deux espèces... Seuls des élevages bien conduits pourront trancher le débat.

\section{BIBLIOGRAPHIE}

Arthur (D. R.). - Ixodes theileri n. sp., with observations on species confused therewithe. Parasitology, 43, 1953, 3-4, 239-245.

Baxks (N.). - A revision of the Ixodoïdea or ticks of the United States. U.S. Dep. of Agr., Bureau of Entom., 1908, 15.

Métıxu (T.). - Méthode pratique pour le transport, l'élevage et la conservation des Ixodoïdes. Ann. de Parasil., 25, 1950, 1-2, 48.

Neumax (G.). - Révision de la famille des Ixodidés; $3^{e}$ mémoire. Mem. Soc. Zool. Fr., 12, 1899, 133-135.

- Notes sur les Ixodidés, II. Arch. de Parasitol., 8, 1904, 454.

Nutrall (G. H. F.). - On four new species of Ixodes. Parasitology, 6, 1913, 131.

Pomerantzev (B. I.). - Fanna, S.S.S.R., 4, 1950, 68 (cité par Arthur, 1953).

(Laboratoire de Parasitologie de la Faculté de Médecine de Bordeaux Professeur: R. Sigalas) 